\title{
Valoración del desarrollo de competencias de los estudiantes mediante mapas perceptuales: Una aplicación en la Facultad de Economía y Empresa de la Universidad de Barcelona ${ }^{1}$ Assessing the development of students' competencies by means of perceptual maps: An application to the Faculty of Economics and Business of the University of Barcelona
}

\author{
Oscar Claveria, Ana María Pérez-Marín
}

\author{
Instituto de Investigación en Economía Aplicada (IREA) \\ Departamento de Econometría, Estadística y Economía Aplicada \\ Universidad de Barcelona \\ Diagonal, 690 \\ oclaveria@ub.edu amperez@ub.edu
}

\begin{abstract}
Resumen
El presente artículo se centra en la generación de mapas perceptuales de los ránkings de las percepciones y las expectativas de los graduados universitarios, los profesores y los empresarios en cuanto al desarrollo de competencias. La información parte de una encuesta realizada entre licenciados y académicos de la Facultad de Economía y Empresa de la Universidad de Barcelona y empresarios catalanes. En primer lugar se jerarquizan las competencias y habilidades desarrolladas durante los estudios universitarios. Los ránkings se combinan para los tres colectivos con el objetivo de proyectar visualmente el posicionamiento de los tres tipos de competencias (genéricas, personales y específicas) en mapas perceptuales generados mediante diferentes tipos de técnicas multivariantes de reducción de la dimensionalidad para datos ordinales (escalamiento multidimensional y componentes principales categóricos). A partir del análisis realizado, se observa un elevado consenso entre los tres colectivos por lo que respecta a la relevancia de las competencias. Los mapas perceptuales permiten captar el grado de similitud entre las competencias analizadas en términos de su posicionamiento. Así, destacan como mejor posicionadas la organización y planificación entre las competencias genéricas; la comunicación oral y escrita entre las personales; y la capacidad de aprendizaje entre las específicas.
\end{abstract}

Palabras clave: Competencias, Universidad, Encuesta, Ranking, Posicionamiento, Mapas perceptuales.

\begin{abstract}
This paper focuses on the generation of visual projections of the ranking of perceptions and expectations of graduate students, teachers and employers regarding the development of competencies by means of perceptual maps. We rank the competencies and working abilities acquired during university education (perceptions) and the ones demanded by the labour market (expectations). Both rankings are combined for each collective in order to position each type of competency (generic, personal and specific). We compare the results obtained by multidimensional scaling and categorical principal components. We observe a high degree of consensus among the different groups regarding the relevance of the competencies. Perceptual maps allow us to visually discriminate between the most and the least valued competencies.
\end{abstract}

Key words: Competencies, University, Surveys, Ranking, Positioning, Perceptual maps

\footnotetext{
${ }^{1}$ Este trabajo es fruto del proyecto REDICE-1001-01, financiado por el Instituto de Ciencias de la Educación de la Universidad de Barcelona. Agradecemos también la colaboración de la Secretaria de Estudiantes, el Servicio de Relaciones Externas y el Decanato de la Facultad de Economía y Empresa de la Universidad de Barcelona y de Fomento del Trabajo. Queremos también agradecer al editor de la revista y a dos evaluadores anónimos por los comentarios y sugerencias realizadas.
} 


\section{Introducción}

El creciente interés sobre el desarrollo de competencias por parte de los estudiantes universitarios se ha traducido en la gestación de una literatura centrada en la evaluación de la adquisición de competencias en el ámbito universitario (Alcañiz, Claveria y Torra, 2016; Heijke, Meng y Ris, 2003; Huber, Fennie y Patterson, 2015, Ismail y Meerah, 2012; Jetté et al., 2010; Martín, Rabadán y Hernández, 2013; Munroe y Westwind, 2009; Olehnovica, Bolgzda y Kravale-Paulina, 2015; Roberson, Carnes y Vice, 2002; Salas, 2003). Mientras que una línea de investigaciones se ha centrado en el encaje entre las competencias y habilidades desarrolladas en la universidad y las exigidas en el mercado laboral (Allen y van der Velden, 2001; Archer y Davison, 2008; Atfield y Purcell, 2010; Green y McIntosh, 2007; Mavromaras et al., 2013; Semeijn et al., 2005; World Economic Forum, 2014), este estudio se centra en generar una proyección visual sobre la valoración que tienen tanto los estudiantes graduados, como los profesores y los empresarios sobre el desarrollo de competencias durante los estudios universitarios.

El propósito esencial que se persigue es plasmar el posicionamiento de los diferentes tipos de competencias (genéricas, personales y específicas), sintetizando simultáneamente las competencias y habilidades desarrolladas durante los estudios universitarios (percepciones) y las que se les exige a los estudiantes graduados para incorporarse en el mercado laboral (expectativas). Para ello se utilizan mapas perceptuales basados en técnicas de reducción de dimensionalidad. La investigación se basa en los datos recabados a través de una encuesta llevada a cabo entre graduados, académicos y empleadores. El presente estudio se centra en la valoración que realizan los encuestados sobre cada competencia. A partir de la jerarquización de las valoraciones medias se sintetiza la información de los tres colectivos analizados y se proyecta en mapas perceptuales generados mediante técnicas multivariantes para información ordinal. Concretamente, se combina el análisis derivado del escalamiento multidimensional (MDS) y de componentes principales categóricos (CATPCA).

En la siguiente sección se describe la base de datos y se presenta los ránkings y la metodología utilizada. En la tercera sección se presentan los mapas perceptuales generados a partir de los ránkings. Finalmente, en las conclusiones se hace una valoración de los resultados obtenidos.

\section{Datos y Metodología}

El presente estudio se centra en la proyección de los ránkings relativos a la valoración de competencias por parte de profesores, estudiantes y empresarios. Dicha valoración se recoge a través de una encuesta llevada a cabo en la Facultad de Economía y Empresa de la Universidad de Barcelona. La población del estudio está constituida por los estudiantes graduados con una experiencia laboral de entre 2 y 5 años, la plantilla de profesores de la facultad, y un conjunto de empresarios de la región. Los graduados cursaron alguna de las titulaciones impartidas en la Facultad: Administración y Dirección de Empresas, Economía, Ciencias Empresariales, Estadística, Ciencias Actuariales y Financieras, Investigación y Técnicas de Mercado, Estudios Inmobiliarios y de la Construcción, y Graduado Tributario y Contable. La información sobre las empresas proviene de la base de datos institucional, donde figuran las empresas empleadoras de titulados en los estudios impartidos en la Facultad, complementándose con información proveniente de la base de datos de Fomento del Trabajo.

Los cuestionarios se distribuyen on-line. Dado que los individuos responden voluntariamente, la muestra obtenida es no probabilística. Esta técnica de muestreo es ampliamente utilizada en este tipo de investigaciones (Grande y Abascal, 2005; McMillan y Schumacher, 2005). Se obtienen 408 respuestas, de las que el $20.3 \%$ corresponde a profesores, el $43.6 \%$ a graduados, y el $36 \%$ a empresarios. Para una descripción detallada de la encuesta y un análisis descriptivo de los datos relativos a las valoraciones de los encuestados ver Alcañiz, Claveria y Riera (2014). 


\begin{tabular}{|c|c|c|c|c|c|c|}
\hline & \multicolumn{2}{|c|}{ Profesores } & \multicolumn{2}{|c|}{ Graduados } & \multicolumn{2}{|c|}{ Empresarios } \\
\hline & Percepción & Expectativa & Percepción & Expectativa & Percepción & Expectativa \\
\hline \multicolumn{7}{|l|}{ Competencias genéricas } \\
\hline Análisis y síntesis & 1 & 1 & 4 & 5 & 7 & 5 \\
\hline Organización y planificación & 7 & 2 & 3 & 1 & 2 & 6 \\
\hline Conocimientos generales & 6 & 10 & 1 & 9 & 9 & 3 \\
\hline Conocimientos específicos & 4 & 8 & 5 & 10 & 8 & 3 \\
\hline Lenguas extranjeras & 10 & 6 & 10 & 8 & 10 & 10 \\
\hline Informática & 9 & 9 & 9 & 2 & 5 & 2 \\
\hline Aplicación conocimientos & 3 & 2 & 8 & 6 & 6 & 7 \\
\hline Resolución de problemas & 2 & 4 & 7 & 3 & 1 & 8 \\
\hline Gestión de la información & 8 & 7 & 6 & 6 & 3 & 1 \\
\hline Trabajo autónomo & 5 & 5 & 2 & 4 & 4 & 9 \\
\hline \multicolumn{7}{|l|}{ Competencias personales } \\
\hline Comunicación oral & 4 & 1 & 2 & 2 & 2 & 1 \\
\hline Comunicación escrita & 1 & 2 & 1 & 4 & 2 & 2 \\
\hline Capacidad crítica & 2 & 4 & 3 & 8 & 5 & 5 \\
\hline Trabajo en equipo & 5 & 3 & 6 & 3 & 1 & 3 \\
\hline Liderazgo & 9 & 8 & 8 & 7 & 8 & 8 \\
\hline Trabajo bajo presión & 6 & 5 & 4 & 1 & 4 & 7 \\
\hline Transmisión de conocimientos & 3 & 7 & 5 & 6 & 6 & 6 \\
\hline Negociación & 8 & 6 & 9 & 5 & 7 & 9 \\
\hline Apreciación diversidad cultural & 7 & 9 & 7 & 10 & 9 & 4 \\
\hline Capacidad de imponer autoridad & 10 & 10 & 10 & 9 & 10 & 10 \\
\hline \multicolumn{7}{|l|}{ Competencias específicas } \\
\hline Adaptación & 4 & 4 & 5 & 1 & 2 & 3 \\
\hline Aprendizaje & 2 & 2 & 1 & 2 & 1 & 1 \\
\hline Creatividad & 6 & 6 & 10 & 8 & 9 & 9 \\
\hline Iniciativa y espíritu emprendedor & 9 & 5 & 7 & 6 & 5 & 7 \\
\hline Autoexigencia & 7 & 8 & 4 & 5 & 3 & 4 \\
\hline Toma de decisiones & 5 & 3 & 6 & 3 & 7 & 10 \\
\hline Razonamiento económico & 3 & 7 & 2 & 8 & 10 & 5 \\
\hline Interpretación de resultados & 1 & 1 & 3 & 3 & 6 & 6 \\
\hline Elaboración de informes & 8 & 9 & 8 & 7 & 8 & 8 \\
\hline Ética empresarial & 10 & 10 & 8 & 10 & 4 & 2 \\
\hline
\end{tabular}

Tabla 1: Ranking de competencias en función de su valor medio según el colectivo

\begin{tabular}{l|l|l}
\hline \multicolumn{1}{c|}{ Competencias genéricas } & \multicolumn{1}{|c}{ Competencias personales } & \multicolumn{1}{c}{ Competencias específicas } \\
\hline Organización y planificación & Comunicación oral & Aprendizaje \\
Análisis y síntesis & Comunicación escrita & Adaptación \\
Resolución de problemas & Trabajo en equipo & Interpretación de resultados \\
Trabajo autónomo & Capacidad crítica & Autoexigencia \\
Gestión de la información & Trabajo bajo presión & Toma de decisiones \\
Aplicación conocimientos & Transmisión de conocimientos & Razonamiento económico \\
Informática & Negociación & Iniciativa y espíritu emprendedor \\
Conocimientos generales & Apreciación diversidad cultural & Ética empresarial \\
Conocimientos específicos & Liderazgo & Creatividad \\
Lenguas extranjeras & Capacidad de imponer autoridad & Elaboración de informes \\
\hline
\end{tabular}

Tabla 2: Listado de competencias ordenadas en función de su valoración media

En la Tabla 1 queda recogida la taxonomía de competencias utilizada en el presente estudio. Esta clasificación parte fundamentalmente de los cuestionarios ICE Tuning (Paganini, 2009). Las competencias se dividen en: genéricas, personales, y específicas. Asimismo, cada uno de los tres colectivos analizados valora mediante una escala Likert de 1 a 6 cada una de las competencias. A 
continuación se calcula la valoración media para cada colectivo y tipo de competencia y se generan los ránkings de competencias que figuran en la Tabla 1.

El carácter ordinal de la información objeto de estudio hace necesaria la utilización de técnicas de análisis multivariante específicas. Ver Hair et al. (2009) y Jolliffe (2002) para un análisis detallado sobre las diferentes técnicas de análisis multivariante. En este estudio se complementan los mapas perceptuales generados mediante MDS con los diagrames de dispersión biespacial resultantes del CATPCA.

Estas técnicas permiten generar representaciones de dimensionalidad reducida que preservan tanta información como es posible de la base de datos original. De esta manera facilitan la detección de patrones subyacentes en la relación existente entre las variables (Chandra y Menezes, 2001). Las técnicas de reducción de la dimensionalidad se han utilizado en diferentes ámbitos: desde análisis sobre la percepción (Phau, Shanka y Dhayan, 2010), estudios sobre la motivación (Park y Yoon, 2009), hasta otras áreas como el diseño de indicadores (Fetscherin y Stephano, 2016). Uno de los ámbitos de mayor aplicación de las técnicas de reducción de la dimensionalidad es en los estudios de segmentación de mercados (Guo et al., 2015; Sinclari-Maragh, Gursoy y Vieregge, 2015; Upchurch, Ellis y Seo, 2004).

El MDS también se conoce como análisis de coordenadas principales (Torgerson, 1952, 1958). Esta técnica permite la generación de proyecciones denominadas mapas perceptuales, que capturan el nivel de similaridad entre individuos basado en la proximidad entre ellos. Para una descripción detallada sobre MDS ver Borg y Groenen (2005) y Fentom y Pearce (1998). En una revisión de la literatura relacionada con la aplicación de MDS, Marcussen (2014) encuentra que uno de los ámbitos de aplicación más frecuente es el posicionamiento (Claveria, 2016; Kim, Kim y Han, 2007; Li et al., 2015; Uysal, Chen y Williams, 2000).

Con el objetivo de analizar las potenciales relaciones de tipo no lineal en los datos de tipo cualitativo, el marco del análisis de componentes principales lineales (Pearson, 1901) se extiende en diferentes direcciones. Guttman (1941) desarrolló una primera extensión para el análisis no lineal. Otras extensiones más recientes, son las relacionadas con la utilización de redes neuronales asociativas (Kramer, 1991), los enfoques kernel (Schölkopf et al., 1998) y los mapas autoorganizados (SOM) (Kohonen, 1982, 2001). Los SOM son redes neuronales artificiales utilizadas para generar proyecciones visuales de los datos que permiten revelar patrones de comportamiento difícilmente observables (Liu y Weisberg, 2005). Otra generalización nolineal de los componentes principales es el CATPCA, conocido también como PCA no lineal, y que recientemente se ha empezado a utilizar en estudios económicos (Claveria et al., 2016; Sarlin y Peltonen, 2013).

El CATPCA fue desarrollado por el Data Theory Scaling System Group, de la Faculty of Social and Behavioral Sciences de la Universidad de Leiden (Linting et al., 2007; Meulman, 2004). Esta técnica presenta diferentes ventajas respecto los Componentes princiaples estándar: por un lado, permite analizar información tanto de tipo ordinal como nominal; por otro, no parte del supuesto que las relaciones entre las variables son lineales. Una ventaja adicional de esta técnica se debe al hecho de que tiende a concentrar mayor variación en los primeros componentes debido a la transformación previa que se realiza de las variables mediante un proceso de cuantificación óptima (De Leeuw y Meulman, 1986).

Linting et al. (2007) presentan una descripción detallada sobre la implementación de CATPCA. Debido a la novedad de la técnica, existen menos aplicaciones de CATPCA que de MDS. Claveria y Poluzzi (2017) realizan una comparativa aplicada de ambas técnicas. Ver Gifi (1990) para un análisis detallado sobre las diferentes técnicas de análisis multivariante nolineal. En este trabajo complementamos los mapas perceptuales de los ránkings de competencias que se presentan en las Tabla 1 con las proyecciones bidimensionales fruto de aplicar CATPCA. 


\section{Resultados}

A continuación se presenta para cada tipo de competencia el mapa perceptual (MDS) y el gráfico de dispersión biespacial (CATPCA) resultante. La metodología seguida se basa en un trabajo reciente de Claveria (2016), y consiste en agregar los ránkings de la Tabla 1 para los tres colectivos con el objetivo de aglutinar toda la información en un conjunto de indicadores sintéticos que mantienen la estructura original de los datos para cada tipo de competencia. Dado que la proyección bidimensional favorece la interpretación de los resultados, ambos tipos de análisis se centran en la representación de los dos primeros componentes. En ambos casos se generan proyecciones de la información original en un espacio de dos dimensiones, por consiguiente la proximidad entre los individuos, que en nuestro caso son los diferentes tipos de competencias, es indicativa de una mayor similitud en términos comparativos.

Así, en la Figura 1 se presenta el análisis para las competencias generales, en la Figura 2 para las competencias personales, y finalmente en la Figura 3 para las competencias específicas. En todos los casos se puede observar como la primera dimensión concentra los mayores valores referentes a las puntuaciones de las diferentes competencias en las dimensiones retenidas. Por un lado, este resultado indica que el indicador sintético correspondiente a la primera dimensión mantiene la estructura ordinal original con un mínimo coste en cuanto a la pérdida de información. Por otro, hace que la segunda dimensión acabe capturando una pequeña proporción del comportamiento de las variables analizadas.

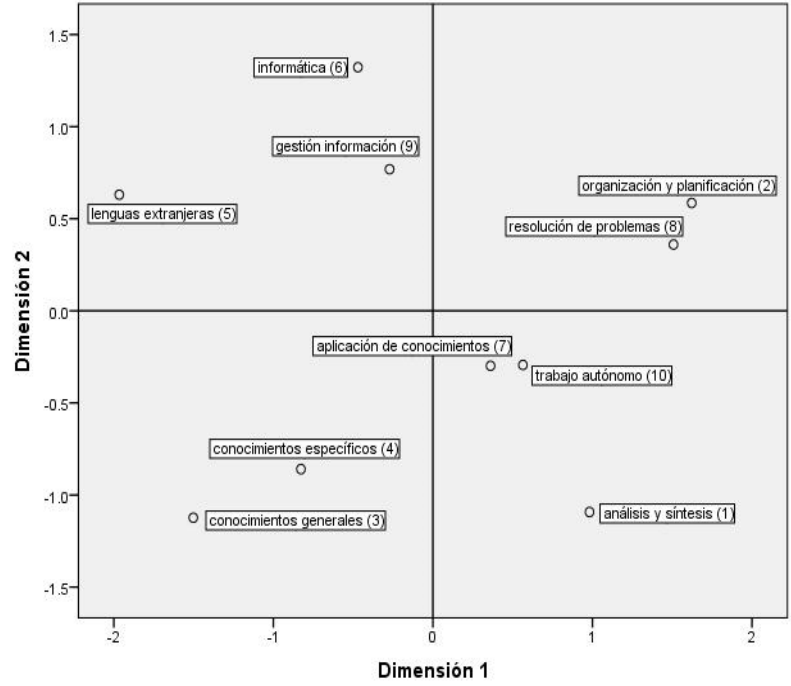

Mapa perceptual

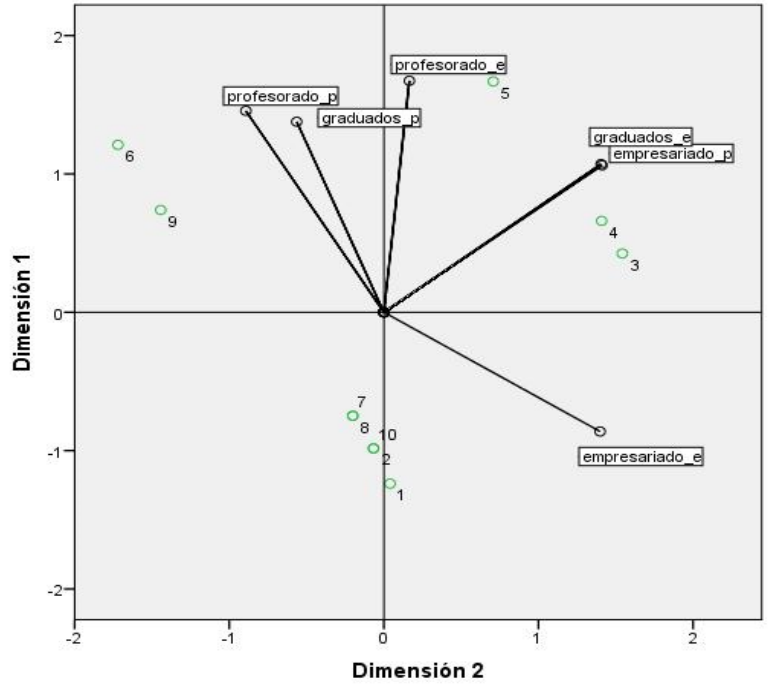

Diagrama de dispersión biespacial

Figura 1: Mapa perceptual y diagrama de dispersión biespacial de las competencias generales

Al comparar los mapas perceptuales con los diagramas de dispersión biespacial se ponen de manifiesto los paralelismos entre el posicionamiento de las competencias obtenido con ambas técnicas. Así, en la Figura 1 se observa como las competencias mejor posicionadas, independientemente del colectivo, son "organización y planificación" y "análisis y síntesis". En ambos casos, las competencias tienen una elevada puntuación en la primera dimensión para el análisis MDS y bajo para el análisis CATPCA, en el cual no está implementada la rotación de los ejes para SPSS. 


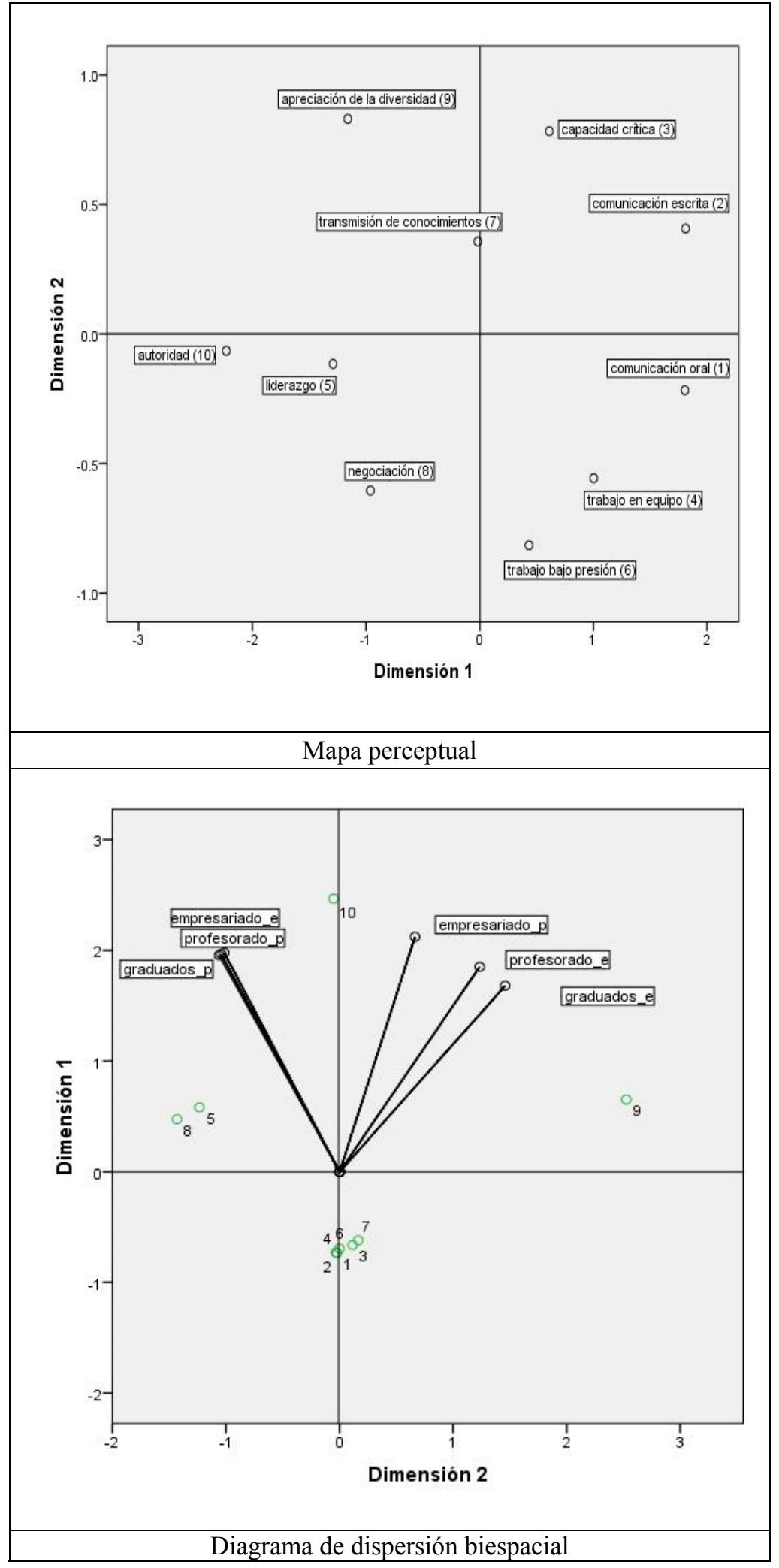

Figura 2: Mapa perceptual y diagrama de dispersión biespacial de las competencias personales 
RIDU Revista d'Innovacio Docent Universitaria 9 (2017) 54-65

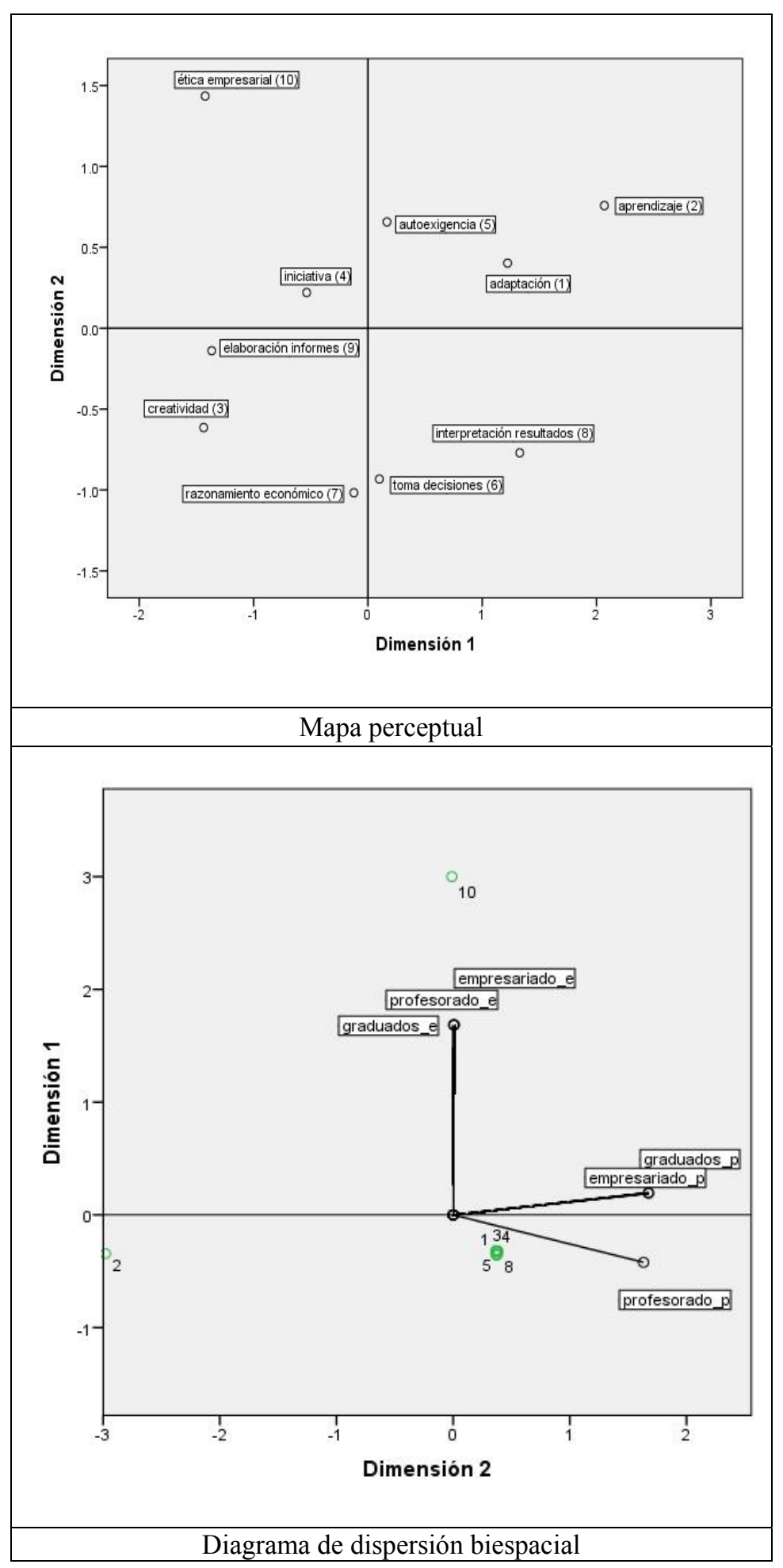

Figura 3: Mapa perceptual y diagrama de dispersión biespacial de las competencias específicas 
Los mapas de dispersión biespacial generados mediante CATPCA, además de proyectar las competencias, solapan adicionalmente los vectores que representan las saturaciones de los componentes ajustadas a los objetos (competencias). Cuanto mayor sea la longitud del vector correspondiente a la expectativa (e) sobre la valoración óptima de cada competencia de cara a la incorporación en el mercado laboral o la percepción ( $\mathrm{p}$ ) respecto al desarrollo efectivamente observado para cada colectivo, mejor será el ajuste. La dirección de los vectores también informa respecto a la relación existente entre las valoraciones efectuadas por cada colectivo analizado. Así, direcciones similares en el mismo sentido, indican una relación positiva; direcciones similares en sentidos opuestos (formando un ángulo de aproximadamente $180^{\circ}$ entre ambos vectores), se corresponden con una relación inversa; finalmente, los vectores que forman aproximadamente un ángulo recto, indican que no existe relación. En este sentido, cabe resaltar como en la Figura 1, las expectativas del empresariado y la percepción del profesorado muestran una fuerte relación inversa.

En la Figura 2 se puede observar como las dos competencias mejor posicionadas independientemente del colectivo analizado son la "comunicación oral" y la "comunicación escrita", por contraposición a "autoridad" y el "liderazgo". Finalmente, en la Figura 3 se observa como el "aprendizaje", que es la competencia mejor valorada, está posicionada al margen del resto de competencias en ambos gráficos, especialmente en el diagrama de dispersión biespacial. Esto es así dado que el CATPCA tiende a polarizar las diferencias entre los objetos proyectados. Dado que el "aprendizaje" es la competencia específica mejor posicionada, con diferencia respecto a la segunda mejor posicionada ("adaptación"), esta diferencia relativa queda amplificada en la proyección. En el diagrama de dispersión biespacial también se puede observar como la jerarquización de las percepciones está muy poco relacionada con la de las expectativas de los tres colectivos analizados (empresarios, graduados y profesores). El hecho de que esta discrepancia sea ligeramente superior entre el profesorado estaría poniendo de manifiesto una visión más crítica en cuanto al desarrollo competencial, la cual vendría condicionada por su directa implicación en el proceso de adquisición de competencias durante los años de universidad.

Estos resultados están en consonancia con los de Alcañiz, Claveria y Riera (2014), quienes detectan la existencia de diferencias significativas entre la percepción sobre las competencias desarrolladas en la universidad y las expectativas sobre el nivel necesario para el desarrollo profesional de los graduados. Los autores también encuentran que esta disparidad de criterio es especialmente acentuada en los empresarios frente a profesores y graduados. Chevalier y Lindley (2009) y Di Pietro y Urwin (2006) también se hacen eco de este desajuste a nivel europeo. No obstante, cabe recordar que el objetivo del presente estudio es puramente descriptivo, y no se realiza inferencia alguna. Las proyecciones ponen de manifiesto las similitudes entre las competencias analizadas de acuerdo con su valoración por los tres colectivos analizados.

\section{Conclusiones}

El objetivo del presente estudio es profundizar sobre el posicionamiento y la valoración de las competencias por parte no solo de la comunidad universitaria, sino también de los empresarios. Así, a través de una encuesta llevada a cabo entre todos los agentes implicados (graduados, docentes universitarios y empresarios) se recoge la información relativa a la valoración de las diferentes competencias, distinguiendo entre la percepción sobre el nivel adquirido durante el período formativo y la expectativa sobre el nivel requerido de cara a la inserción laboral.

Con el objetivo de posicionar las diferentes competencias analizadas, se realiza un ránking de éstas para cada uno de los colectivos analizados. A continuación se aplican diferentes técnicas multivariantes para de reducción de la dimensionalidad para generar proyecciones en dos dimensiones que sinteticen toda la información manteniendo la estructura original de los datos. Dada la naturaleza ordinal de las variables analizadas, se ha utilizado el análisis de escalamiento multidimensional para generar mapas perceptuales 
que se comparan con los diagramas de dispersión biespacial resultantes del análisis de componentes principales categóricos.

Mediante la proyección de las dos primeras dimensiones retenidas se detectan diferencias notables por lo que respecta al posicionamiento en cuanto a la valoración de las diferentes competencias. Por lo que respecta a las competencias genéricas, la "organización y la planificación", seguida de la capacidad de "análisis y síntesis", son las competencias mejor posicionadas independientemente del colectivo analizado. La "comunicación oral" y "escrita" son las competencias personales mejor posicionadas, mientras que la capacidad de "aprendizaje" es la competencia específica mejor posicionada. En el extremo opuesto se encuentran las "lenguas extranjeras", la capacidad de imponer "autoridad" y de "elaborar informes" respectivamente.

Adicionalmente se ha detectado la existencia de diferencias significativas entre la percepción sobre las competencias desarrolladas en la universidad por parte de los profesores y las expectativas sobre el nivel necesario para el desarrollo profesional de los graduados, especialmente entre el colectivo de empresarios. Esta disparidad de criterio es especialmente relevante entre los empresarios, y podría estar poniendo de relieve la potencial sobreeducación de los graduados.

Con el objetivo de ahondar sobre esta cuestión se plantean diferentes líneas de investigación futuras. Por un lado, un análisis comparativo entre diferentes áreas de conocimiento haría posible examinar las potenciales diferencias sectoriales y aumentar el tamaño muestral. Por otro lado, la extensión del estudio a otras universidades españolas y extranjeras permitiría analizar las diferencias existentes a nivel regional e internacional.

\section{Referencias}

Alcañiz, M., Claveria, O., Riera, C. (2014) Competencias en educación superior desde tres perspectivas diferentes: estudiantes, empleadores y académicos. Revista Iberoamericana de Educación, 66(2), pp. 1-19.

Alcañiz, M., Claveria, O., Torra, S. (2016) Posicionament multivariant de les expectatives d'estudiants graduats, professors i empresaris mitjançant components principals categòrics. Revista d'Innovació Docent Universitària 8, pp. $77-85$.

Allen, J., van der Velden, R. (2001) Educational mismatches versus skill mismatches: effects on wages, job satisfaction and on-the-job search. Oxford Economic Papers, 3, pp. 434-452.

Archer, W., Davison, J. (2008) Graduate Employability: What do employers think and want? Council for Industry and Higher Education (CIHE), London.

Atfield, G., Purcell, K. (2010) Graduate labour market supply and demand: Final year students' perceptions of the skills they have to offer and the skills employers seek. Working Paper 4, Institute for Employment Research, University of Warwick, Warwick.

Borg, I., Groenen, P.J.F. (2005) Modern multidimensional scaling: Theory and applications (2nd Ed.). SpringerVerlag, New York.

Chandra, S., Menezes, D. (2001) Applications of multivariate analysis in international tourism research: The marketing strategy perspective of NTOs. Journal of Economic and Social Research, 3(1), pp. 77-98.

Chevalier, A., Lindley, J. (2009) Overeducation and the skills of UK graduates. Journal of the Royal Statistical Society: Series A, 172, pp. 307-337.

Claveria, O. (2016) Positioning emerging markets using tourism and economic indicators. Journal of Hospitality and Tourism Management, 29, pp. 143-153. 
Claveria, O., Monte, E., Torra, S. (2016) A self-organizing map analysis of survey-based agents' expectations before impending shocks for model selection: The case of the 2008 financial crisis. International Economics, 146, pp. $40-58$.

Claveria, O., Poluzzi, A. (2017) Positioning and clustering of the world's top tourist destinations by means of dimensionality reduction techniques for categorical data. Journal of Destination Marketing \& Management. In Press.

De Leeuw, J., Meulman, J.J. (1986) Principal component analysis and restricted multidimensional scaling. En: W. Gaul y M. Schader (Eds.) Classification as a Tool of Research. Amsterdam: North-Holland.

Di Pietro, G., Urwin, P. (2006) Education and skills mismatch in the Italian graduate labour market. Applied Economics, 38(1), pp. 79-93.

Fentom, M., Pearce, P. (1988) Multidimensional scaling and tourism research. Annals of Tourism Research, 15, pp. 236-254.

Fetscherin, M., Stephano, R.M. (2016) The medical tourism index: Scale development and validation. Tourism Management, 52(1), pp. 539-556.

Gifi, A. (1990) Nonlinear multivariate analysis. Wiley, Chichester, England.

Grande, I., Abascal, E. (2005) Análisis de encuestas. ESIC, Madrid.

Green, F., McIntosh, S. (2007) Is there a genuine under-utilization of skills amongst the over-qualified? Applied Economics, 39(4), pp. 427-439.

Guo, Y., Guillet, B.D., Kucukusta, D., Law, R. (2015) Segmenting hotel customers based on rate fences using conjoint and cluster analyses. Journal of Travel \& Tourism Marketing, 32(7), pp. 835-851.

Guttman, L. (1941) The quantification of a class of attributes: A theory and method of scale construction. En: P. Horst (Ed.) The prediction of personal adjustment. Social Science Research Council, New York.

Hair, J.F., Black, W.C., Babin, B.J., Anderson, R.E. (2009) Multivariate data analysis (7th Ed.). Prentice Hall: Upper Saddle River, NJ.

Heijke, H., Meng, C., Ris, C. (2003) Fitting to the job: the role of generic and vocational competencies in adjustment and performance. Labour Economics, 10(2), pp. 215-229.

Huber, R.L.B., Fennie, K., Patterson, H. (2015) Competencies for master and doctoral students in epidemiology: what is important, what is unimportant, and where is there room for improvement? Annals of Epidemiology, 25, pp. 466-468.

Isamail, R., Meerah, S.M. (2012) Evaluating the research competencies of doctoral students. Procedia - Social and Behavioral Sciences, 59, pp. 244-247.

Jetté, S., Tribble, D.S.C., Gagnon, J., Mathieu, L. (2010) Nursing students' perceptions of their resources toward the development of competencies in nursing informatics. Nurse Education Today, 30, pp. 742-746.

Jolliffe, I.T. (2002) Principal component analysis (2nd Ed.). Springer Series in Statistics.

Kim, D.J., Kim, W.G., Han, J.S. (2007) A perceptual mapping of online travel agencies and preference attributes. Tourism Management, 28(2), pp. 591-603.

King, C., Chen, N., Funk, D.C. (2015) Exploring destination image decay. Journal of Hospitality and Tourism Research, 39 (1), pp. 3-31. 
Kohonen, T. (1982) Self-organized formation of topologically correct feature maps. Biological Cybernetics, 43(1), pp. 59-69.

Kohonen, T. (2001) Self-organizing maps. Springer, Berlin.

Kramer, M.A. (1991) Nonlinear principal component analysis using autoassociative neural networks. $A I C h E$ Journal, 37(2), pp. 233-243.

Li, X.R., Cheng, C.K., Kim, H., Li, X. (2015) Positioning USA in the Chinese outbound travel market. Journal of Hospitality and Tourism Research, 39(1), pp. 75-104.

Linting, M., Meulman, J.J., Groenen, P.J.F., Van der Koojj, A.J. (2007) Nonlinear principal components analysis: Introduction and application. Psychological Methods, 12(3), pp. 336-358.

Liu, Y., Weisberg, R.H. (2005) Patterns of ocean current variability on the west Florida shelf using the selforganizing map. Journal of Geophysical Research, 110(C6), pp. 1-12.

Marcussen, C.H. (2014) Multidimensional scaling in tourism literature. Tourism Management Perspectives, 12, pp. $31-40$.

Martín, M., Rabadán, A.B., Hernández, J. (2013) Desajustes entre formación y empleo en el ámbito de las Enseñanzas Técnicas universitarias: la visión de los empleadores de la Comunidad de Madrid. Revista de Educación, 360(1), pp. 244-267.

Mavromaras, K., McGuinness, S., O'Leary, N., Sloane, P., Wei, Z. (2013) Job Mismatches and Labour Market Outcomes: Panel Evidence on University Graduates. Economic Record, 89, pp. 382-395.

McMillan, J.H., Schumacher, S. (2005) Investigación educativa. Pearson Addison Wesley, Madrid.

Meulman, J.J., Heiser, W.J., SPSS. (2004) SPSS Categories 13.0. Chicago, Illinois: SPSS Inc.

Munroe, T., Westwind, M. (2009) What makes Silicon Valley tick? The ecology of innovation at work. Nova Vista Publishing, New York.

Olehnovica, E., Bolgzda, I., Kravale-Paulina, M. (2015) Individual potential of doctoral students: Structure of research competences and self-assessment. Procedia - Social and Behavioral Sciences, 174, pp. 3557-3564.

Paganini, R. (2009) Una introducción a Tuning Educational Structures in Europe. La contribución de las universidades al proceso de Bolonia. Publicaciones de la Universidad de Deusto, Bilbao.

Park, D.B., Yoon, Y.S. (2009) Segmentation by motivation in rural tourism: A Korean case study. Tourism Management, 30(1), pp. 99-108.

Pearson, K. (1901). On lines and planes of closest fit to systems of points in space. Philosophical Magazine Series 6, 2(11), pp. 559-572.

Phau, I., Shanka, T., Dhayan, N. (2010) Destination image and choice intention of university student travellers to Mauritius. International Journal of Contemporary Hospitality Management, 22(5), pp. 758-764.

Roberson, M.T., Carnes, L.W., Vice, J.P. (2002) Defining and measuring student competencies: A content validation approach for business program outcome assessment. Delta Pi Epsilon Journal 44 (1), pp. 13-24.

Salas, M. (2003) Educación superior y mercado de trabajo. Grupo Editorial Universitario, Granada.

Sarlin, P., Peltonen, T.A. (2013) Mapping the state of financial stability. Journal of International Financial Markets, Institutions \& Money, 26, pp. 46-76. 
Schölkopf, B., Smola, A.J., Müller, K. (1988) Nonlinear component analysis as a kernel eigenvalue problem. Neural Computation, 10(5), pp. 1299-1319.

Semeijen, J.H., van der Velden, R., Heijke, H., van der Vleuten, C., Boshuizen, H.P.A. (2008) Competence indicators in academic education and early labour market success of graduates in health sciences. Research Centre for Education and the Labour Market, Maastricht University, Maastricht.

Sinclari-Maragh, G., Gursoy, D., Vieregge, M. (2015) Residents’ perceptions toward tourism development: A factor-cluster approach. Journal of Destination Marketing \& Management, 4(1), pp. 36-45.

Torgerson, W.S. (1952) Multidimensional scaling: I. Theory and method. Psychometrika, 17(4), pp. 401-419.

Torgerson, W.S. (1958) Theory \& methods of scaling. Wiley, Oxford, England.

Upchurch, R.S, Ellis, T., Seo, J. (2004) Applying the hierarchical cluster analysis procedure upon the process of yield management-A comparative study. Journal of Travel \& Tourism Marketing, 16(4), pp. 47-58.

Uysal, M., Chen, J., Williams, D.R. (2000) Increasing state market share through a regional positioning. Tourism Management, 21(1), pp. 89-96.

World Economic Forum (2014) Matching skills and labour market needs. Paper prepared by the Global Agenda Council on Employment, Davos. 